\title{
Enhancing cisplatin sensitivity in MCF-7 human breast cancer cells by down-regulation of Bcl-2 and cyclin D1
}

\author{
CHRISTINA WESTMOSE YDE and OLAF-GEORG ISSINGER
}

\author{
Institute of Biochemistry and Molecular Biology, University of Southern Denmark, Odense, Denmark
}

Received June 13, 2006; Accepted July 25, 2006

\begin{abstract}
MCF-7 human breast cancer cells are relatively resistant to cisplatin treatment compared to other breast cancer cell lines. In order to identify possible targets for sensitizing the breast cancer cells to cisplatin treatment protein expression levels and the phosphorylation status of 27 different signaling proteins were examined. MCF-7 cells expressed high levels of anti-apoptotic Bcl-2 protein relative to more cisplatin sensitive breast cancer cells. After cisplatin treatment a decrease in cyclin D1 was seen in all the breast cancer cells studied. Therefore, Bcl-2 and cyclin D1 were chosen as putative targets for increasing cell death and growth arrest induced by cisplatin, thereby enhancing the drug sensitivity in MCF-7. RNA interference, using Bcl-2- and cyclin D1siRNAs sensitized MCF-7 cells to cisplatin treatment and by simultaneous knockdown of both Bcl-2 and cyclin D1 further sensitization was seen. This shows the potential of targeting both apoptotic- and cell cycle-regulating pathways to enhance the effect of chemotherapy.
\end{abstract}

\section{Introduction}

Cisplatin (cis-diamminedichloroplatinumII) is a widely used chemotherapeutic compound, known to cause DNA damage by alkylating DNA molecules, causing both inter- and intrastrand crosslinks. Cisplatin activates multiple intracellular pathways including those involving checkpoint kinases, tumor suppressor p53, mitogen-activated protein kinases (MAPKs), cell cycle regulating proteins and caspases (1). These pathways are in addition regulated by other cellular proteins including protein kinases Akt (2), protein kinase C (PKC) (3) and CK2 (formerly called casein kinase 2) (4). The outcome of cisplatin-induced signaling is usually cell cycle arrest, DNA repair and eventually apoptosis and cell death (1) although, the exact processes responsible for cisplatin-

Correspondence to: Dr Olaf-Georg Issinger, Institute of Biochemistry and Molecular Biology, University of Southern Denmark, Campusvej 55, DK-5230 Odense M, Denmark

E-mail: ogi@bmb.sdu.dk

Key words: breast cancer, cisplatin, apoptosis, Bcl-2, cyclin D1 induced cell death and the processes involved in regulating cisplatin sensitivity are still being studied. A major disadvantage to the use of compounds such as cisplatin is the severe dosedependent side effects and in many cases tumors become resistant to the applied treatment. One approach for improving the overall outcome of cancer treatment would be to treat patients simultaneously with compounds enhancing the effect of the apoptotic drug.

Anti-apoptotic protein Bcl-2 is involved in release of cytochrome $\mathrm{C}$ and other apoptosis-promoting factors from the mitochondria and in turn activation of caspases. Bcl-2 has been suggested to be a regulator of chemosensitivity in cancer where at least in some cell lines it has been shown to suppress cell death induced by cytotoxic compounds such as cisplatin, etoposide and doxorubicin $(5,6)$. Bcl-2 antisense oligodeoxynucleotides (also known as G3139, Genasense or Oblimersen), are currently used in clinical trials $(7,8)$ however, the potential of Bcl-2 as anti-cancer target has been questioned e.g. by reports on lack of chemo-sensitization by $\mathrm{Bcl}-2$ downregulation in some cell lines (9).

Cyclin D1 is one of the most frequently overexpressed proteins in breast cancer (10). The proto-oncogenic potential of cyclin D1 has partly been associated with its regulation of cell cycle progression. D-type cyclins associate with cyclindependent kinases (CDKs) 4 and 6 to stimulate phosphorylation of $\mathrm{Rb}$ protein, dissociating $\mathrm{Rb}$ from $\mathrm{E} 2 \mathrm{~F}$ transcription factor resulting in E2F-dependent transcription and G1-S phase transition (11). Investigations of cyclin D1 and E knockout mice revealed high redundancy of the cyclins for normal tissue development (12). Cyclin D1 knockout mice were viable with only focal developmental defects, such as hypoplastic retinopathy and defective breast development during pregnancy, largely confined to the retina and breast tissue (13-15), and mouse embryonic fibroblasts lacking cyclin D1 showed increased UV-induced apoptosis (16). Cyclin D1 has also been shown to display CDK-independent functions, interacting with several transcription factors and transcriptional co-regulators, such as estrogen receptor $\alpha$ and histone acetylase p300/CBP (cAMP response elementbinding protein-binding protein) (17). All these data indicate that the role played by cyclin D1 seems to be much more complex than previously assumed; involving cyclin D1 not only in cell cycle control but also in pathways regulating cellular differentiation and survival. The role of cyclin D1 in affecting cisplatin sensitivity has been studied in various human cancer cell lines, including pancreatic and head and 
neck squamous carcinoma cell lines (18-20) but the relationship between cyclin D1 and cisplatin resistance in breast cancer has not been extensively studied.

In the present work, we show that Bcl-2 and cyclin D1 are both targets for enhancing the apoptotic potential of cisplatin in MCF-7 breast cancer cells and we demonstrate that the most efficient killing of MCF-7 cells is observed when combining cisplatin with down-regulation of both Bcl-2 and cyclin D1.

\section{Materials and methods}

Reagents. Cisplatin and propidium iodide were from Sigma-Aldrich (St. Louis, MO); Complete protease inhibitor cocktail and WST-1 proliferation reagent were from Roche (Indianapolis, IN); protein assay reagent was from Bio-Rad Laboratories (Hercules, CA); SMARTpool Bcl-2 siRNA and scrambled sequence siRNA (GCUCAGAUCAAUACG GAGAdTdT) were from Dharmacon (Lafayette, CO), cyclin D1 siRNA pool was from Santa Cruz Biotechnology (Santa Cruz, CA) and cell culture media, Lipofectamine 2000, and SYTOX green nucleic acid stain were from Invitrogen (Carlsbad, CA). Primary antibodies directed against: Akt2 (F-7), ASK1 (F-9), Bcl-2 (C-2), cyclin D1 (M-20), cyclin E (C-19), estrogen receptor $\alpha$ (D-12), JNK1/2 (D-2), MDM2 (Smp14), p38 $\alpha$ (H-147), PKA (C-20), PKC (A-3), and PKCס (C-20) were purchased from Santa Cruz Biotechnology; Akt1 (610860), GSK3ß (610201), and PKC $\alpha$ (610107) were from BD Transduction Laboratories (San Diego, CA); phosphoT308-Akt (9275), procaspase-3 (3G2), procaspase-9 (C-9), ERK1/2 (9102), phospho-T202/Y204-ERK1/2 (197G2), phospho-S9-GSK3ß (9336), MEK1 (H-8), MKK4 (9152), phospho-T180/Y182-p38 (9211), phospho-ßIIS660-PKC (9371), and $\mathrm{PKC} \zeta(\mathrm{H}-1)$ were from Cell Signaling Technology (Beverly, MA); PTEN (07-016) was from Upstate Biotechnology (Lake Placid, NY); CK2 $\alpha / \alpha$ ' (1AD9), CK2ß (6D5), p53 (Ab-2), p21/Waf1 (Ab-5), and phospho-S15-p53 (Ab-3) were from Calbiochem, PARP (6639GR) was from Pharmingen (San Diego, CA); ß-actin (AC-15) was from Sigma-Aldrich. Anti-CK2 $\alpha$ ' antibody was raised in rabbit against the peptide ${ }^{334}$ SQPCADNAVLSSGLTAAR ${ }^{350}$ derived from human CK2 $\alpha$ '. Secondary antibodies, alkaline phosphatase conjugated goat anti-mouse IgG, goat anti rabbit IgG or rabbit anti-goat IgG, were from Jackson ImmunoResearch Laboratories (Bar Harbor, $\mathrm{ME})$.

Cell culture. Human breast carcinoma cell lines MCF-7, MDA-MB-231, MDA-MB-435, HCC-1937, and CAL-148 were from DSMZ (Braunschweig, Germany). Cells were grown in the presence of $10 \% \mathrm{FBS}$, penicillin (100 units $/ \mathrm{ml}$ ) and streptomycin $(100 \mu \mathrm{g} / \mathrm{ml})$. MCF-7 cells were cultured in RPMI-1640 supplied with $1 \%$ non-essential amino acids. MDA-MB-231 and MDA-MB-435 were cultured in Leibovitz's L-15 medium with GlutaMAX I. Medium for MDA-MB-435 was in addition supplied with $10 \mu \mathrm{g} / \mathrm{ml}$ human insulin. HCC-1937 cells were cultured in RPMI-1640. CAL-148 cells were cultured in Dulbecco's modified Eagle's medium (D-MEM) with GlutaMAX I, $4500 \mathrm{mg} / \mathrm{l}$ D-glucose and $1 \mathrm{mM}$ sodium pyruvate and supplied with $10 \mathrm{ng} / \mathrm{ml}$ human epidermal growth factor.
Cisplatin treatment and cell viability test. Cells were seeded at $30-50 \%$ confluence and left overnight before addition of cisplatin for $24 \mathrm{~h}$. In order to measure cell viability, WST-1 reagent was added to the cells according to the manufacturer's instructions. Conversion of WST-1 to formazan by mitochondrial dehydrogenases was measured in a microtiter plate reader (VERSAMAX, Molecular Devices Ltd).

Protein extraction and Western blotting. Cells were harvested and protein extracts were made by lysing cells in buffer, containing $50 \mathrm{mM}$ Tris- $\mathrm{HCl}, \mathrm{pH} 7.5,150 \mathrm{mM} \mathrm{NaCl}$, $1 \%$ Triton X-100, 10\% glycerol, $1 \mathrm{mM}$ DTT, $30 \mathrm{mM} \mathrm{NaPP}_{\mathrm{i}}$, and $10 \mathrm{mM} \mathrm{NaF}$, supplied with $1 \mathrm{mM}$ activated $\mathrm{Na}_{3} \mathrm{VO}_{4}$ and $1 \mathrm{X}$ protease inhibitor cocktail. Determination of protein concentration was performed using the Bio-Rad protein assay and bovine serum albumin as standard. Proteins were separated by SDS-PAGE and transfered to PVDF membranes by electroblotting. Membranes were blocked with PBS containing $0.2 \%$ casein and $0.1 \%$ Tween-20 followed by incubation with primary and secondary antibodies. Detection of antibody-protein complexes was done using the chemiluminescence CDP-star (Tropix, Bedford, CA).

RNA interference. MCF-7 cells were seeded at 30\% confluence in growth medium without antibiotic. Transfection was done according to the manufacturer's instructions, using Bcl-2 siRNA, cyclin D1 siRNA and scrambled sequence siRNA at final concentrations of $100 \mathrm{nM}, 40 \mathrm{nM}$ and $100 \mathrm{nM}$, respectively. Lipofectamine 2000 was used as transfection reagent. After 24 and $48 \mathrm{~h}$ cells were harvested for Western blot analysis for detection of Bcl-2 and cyclin D1. For cisplatin experiments cells were treated with siRNA for $48 \mathrm{~h}$; for the last $24 \mathrm{~h}$ cisplatin was added as indicated. Control cells (mock) were treated with transfection reagent for $48 \mathrm{~h}$. At least two independent experiments were performed with similar results.

Flow cytometry. For determination of cell death, cells were incubated with SYTOX green nucleic acid stain, harvested and resuspended in PBS containing 1\% FBS. For cell cycle analysis, cells were fixed in $70 \%$ ethanol overnight at $-20^{\circ} \mathrm{C}$ and stained with propidium iodide $(20 \mu \mathrm{g} / \mathrm{ml})$ and RNaseA $(10 \mu \mathrm{g} / \mathrm{ml})$. All flow cytometric analyses were done using a FACSCalibur (Becton-Dickinson, Franklin Lakes, NJ) flow cytometer. The acquired data were analyzed using the Cell Quest Pro software. For each measurement 10,000 cells were analyzed.

\section{Results}

$M C F-7$ is relatively resistant to cisplatin treatment compared to other breast cancer cell lines. The response to cisplatin treatment was examined in five human breast cancer cell lines by treating the cells with different concentrations of cisplatin for $24 \mathrm{~h}$ and measuring cell viability (Fig. 1A). Cisplatin at $80 \mu \mathrm{M}$ was required to reduce the viability of MCF-7 and MDA-MB-231 cells to $<50 \%$ while cisplatin concentrations at $20 \mu \mathrm{M}$ led to a comparable $50 \%$ reduction in cell viability of MDA-MB-435, HCC-1937 and CAL-148 cells (Fig. 1A). Apoptosis was measured by detection of 
A

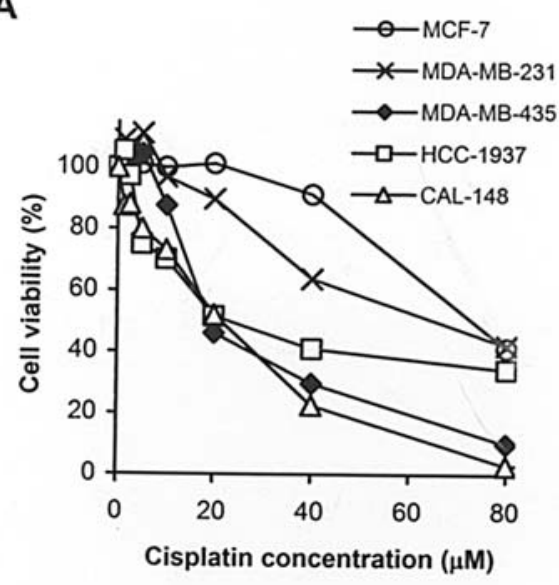

B
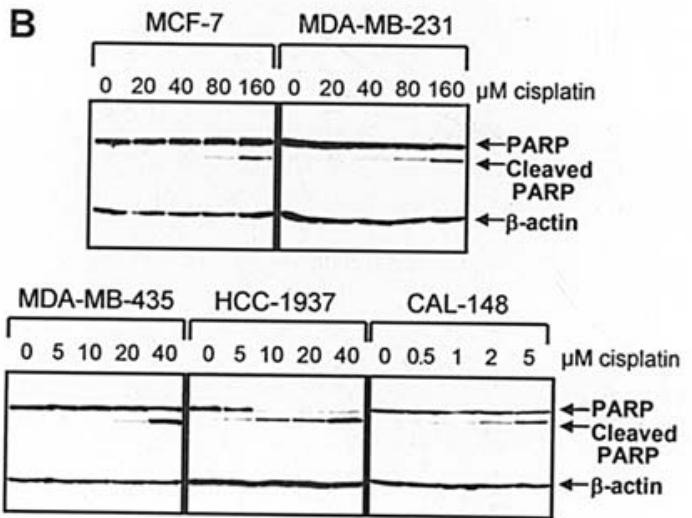

Figure 1. Cisplatin-induced cell death in breast cancer cell lines. Cells were treated for $24 \mathrm{~h}$ with varying concentrations of cisplatin. (A) Conversion of WST-1 to formazan by mitochondrial dehydrogenases as an indication of cell viability was measured (four replicates). (B) Apoptosis induction was measured by PARP cleavage as seen on the Western blots. $\beta$-actin was used as a loading control.

poly-ADP-ribose-polymerase (PARP) cleavage (Fig. 1B). The degree of cisplatin sensitivity observed in the viability test was confirmed, since high concentrations of cisplatin (80-160 $\mu \mathrm{M})$ were required to induce cleavage of PARP in the case of MCF-7 and MDA-MB-231 cells. Cleavage of PARP was induced in MDA-MB-435 cells at 20-40 $\mu \mathrm{M}$ cisplatin and in HCC-1937 and CAL-148 cells at only 2-5 $\mu \mathrm{M}$ of cisplatin (Fig. 1B). Hence, MCF-7 cells and also MDA-MB-231 cells are considered to be relatively resistant to cisplatin treatment compared to the three other human breast cancer cell lines in respect to the cisplatin concentration inducing cell death and apoptosis.

Expression of $\mathrm{Bcl}-2$ protein correlates with cisplatin sensitivity in selected breast cancer cell lines. In order to identify potential targets for enhancing the effect of cisplatin in MCF-7 cells, a comparative study was done including the same breast cancer cell lines used for comparison of cisplatin sensitivity. The levels of 27 different signaling proteins were analyzed before (Fig. 2) and after (Fig. 3) treatment with cisplatin. Cisplatin concentrations, inducing a similar degree of apoptosis in the five cell lines, were chosen as indicated in Fig. 3.

Several interesting proteins could have been selected from the analysis (Fig. 2), however we found that the expression of

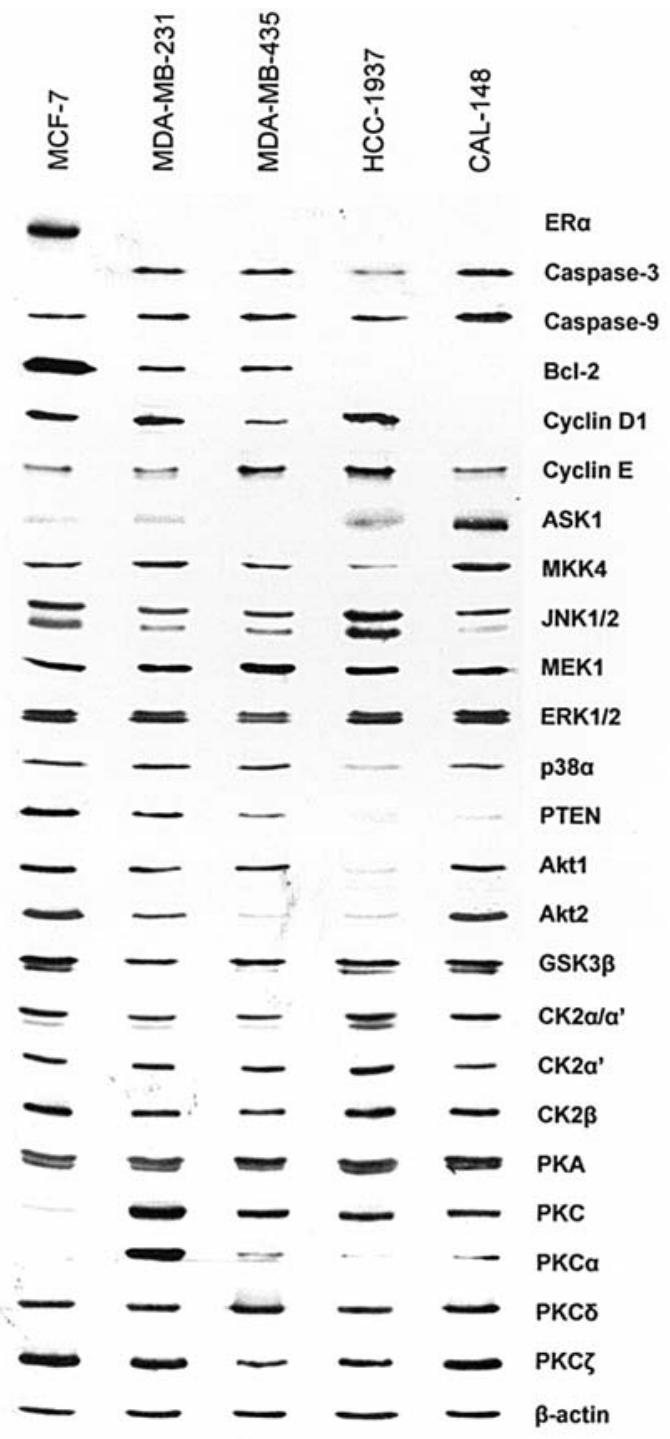

Figure 2. Protein profiling of human breast cancer cell lines. Western blot analysis of signaling proteins was performed as described in the Materials and methods. For all Western blots B-actin was used as control for equal loading and one representative $\beta$-actin blot is shown. JNK1/2: the upper band is JNK2, the lower is JNK1. ERK1/2: the upper band is ERK1, the lower is ERK2. CK2 $\alpha / \alpha^{\prime}$ : the upper band is CK2 $\alpha$, the lower is CK2 $\alpha$ '.

estrogen receptor $\alpha(\mathrm{ER} \alpha)$, caspase-3, Bcl-2, PTEN, protein kinase $\mathrm{C} \alpha(\mathrm{PKC} \alpha)$ and cyclin D1 were of particular interest in respect to cisplatin response: estrogen receptor (ER) status has previously been evaluated as a predictive marker of cisplatin responsiveness and a possible correlation between expression of estrogen receptor and cisplatin resistance was described (21). MCF-7 cells expressed ER $\alpha$, however, MDA-MB-231 cells, which required comparable cisplatin concentrations for induction of cell death did not express detectable ER levels (Fig. 2). Therefore, even though it could not be ruled out that ER $\alpha$ expression could potentially play a role in the cisplatin response in MCF-7 cells, expression of ER alone would not sufficiently explain the differences in cisplatin sensitivity of the cell lines investigated here and no further studies were done on estrogen receptor $\alpha$ expression in this work.

MCF-7 cells harbor a deletion of $47 \mathrm{~kb}$ within the CASP-3 gene encoding procaspase-3 (22) and this was reflected by a 


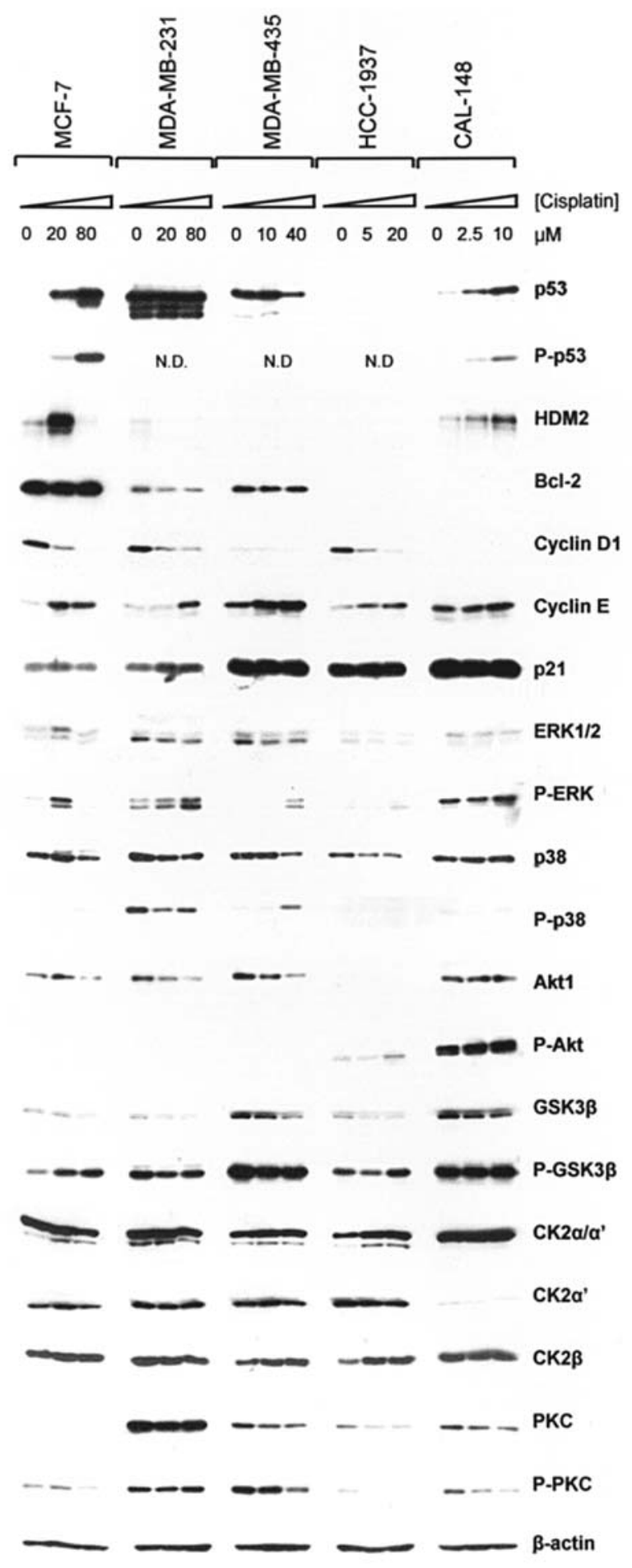

Figure 3. Protein changes upon cisplatin treatment of breast cancer cell lines. Cell were treated with varying concentrations of cisplatin and Western blot analysis of signaling proteins was done as described in the Materials and methods. Primary antibodies are indicated. Residues recognized by phospho-antibodies are: Ser15 (P-p53), Thr202/Tyr204 (P-ERK1/2), Thr180/ Tyr182 (P-p38), Thr308 (P-Akt), Ser9 (P-GSK3ß) and Ser660 (P-PKC). For all blots $B$-actin was used as control for equal loading and one representative ß-actin blot is shown. N.D, not determined.

complete loss of procaspase-3 expression in MCF-7 (Fig. 2). The other four cell lines all expressed procaspase-3. Hence, it seemed that the impaired caspase pathway in MCF-7 cells could be important in respect to the relative resistance of the cell line to cisplatin-induced cells death, however, as we

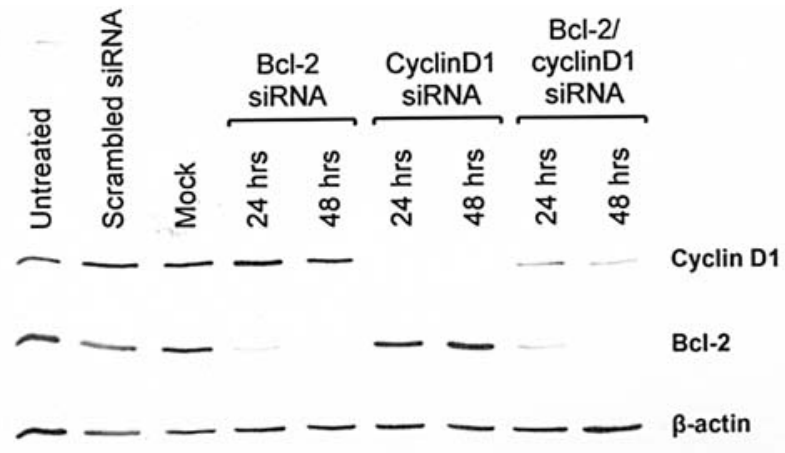

Figure 4. RNA interference of Bcl-2 and cyclin D1 in MCF-7. Cells were transfected with Bcl-2 siRNA (100 nM), cyclin D1 siRNA (40 nM) or Bcl-2/ cyclinD1 siRNA $(100 \mathrm{nM} / 40 \mathrm{nM})$ for 24 and $48 \mathrm{~h}$. As control cell were transfected with scrambled sequence negative control siRNA (100 nM) or with transfection reagent alone (mock) for $48 \mathrm{~h}$. The protein levels of Bcl-2 and cyclin D1 protein were analyzed by Western blotting. B-actin was used as loading control.

wanted to enhance cisplatin sensitivity in MCF-7 by an RNA interference strategy caspase-3 was not selected as a target for our further studies.

The anti-apoptotic protein $\mathrm{Bcl}-2$ was expressed at very high levels in MCF-7 cells and at somewhat lower levels in MDA-MB-231 and MDA-MB-435 cells but was not expressed at detectable levels in HCC-1937 nor in CAL-148 cells (Fig. 2). This was largely in concordance with the cisplatin sensitivities observed for the five breast cancer cell lines (Fig. 1). Therefore, Bcl-2 was chosen as a target for RNA interference studies in $\mathrm{MCF}-7$ cells.

Cyclin D1 expression was interesting because the cell line which was most sensitive to cisplatin, CAL-148, expressed only very low levels of cyclin D1 compared to the other cell lines. Therefore, low cellular levels of cyclin D1 could potentially contribute to increased cisplatin sensitivity, although the other sensitive cell line, i.e. HCC-1937, does not show low levels of cyclin D1.

In the five cell lines studied here it was seen that the cell lines which were most resistant to cisplatin treatment expressed the most PTEN protein (Fig. 2). Several studies have shown that overexpression of PTEN sensitized cancer cells to drug-induced apoptosis $(23,24)$. On the basis of this it was not expected that down-regulation of PTEN would result in increased sensitivity towards cisplatin treatment in MCF-7 cells and PTEN was therefore not chosen for further RNA interference studies.

MCF-7 expressed barely detectable levels of PKC $\alpha$ compared to the other cells lines, where especially MDA-MB231 expressed very hich PKC $\alpha$ levels (Fig. 2). High activity of PKC has been associated with resistance to cisplatin-induced apoptosis in ovarian cancer, small lung carcinoma cells and HeLa cells $(25,26)$. Since MCF-7 already expressed very low levels of $\mathrm{PKC} \alpha$, this kinase was not chosen as a target for RNA interference studies in MCF-7 cells

Several other proteins from the MAPK-pathway, Akt signaling pathway and various protein kinases were included in the Western blot screening (Fig. 2) but were not selected for further studies in the present work. Therefore, the expression pattern of these proteins will not be commented on further in the text. 


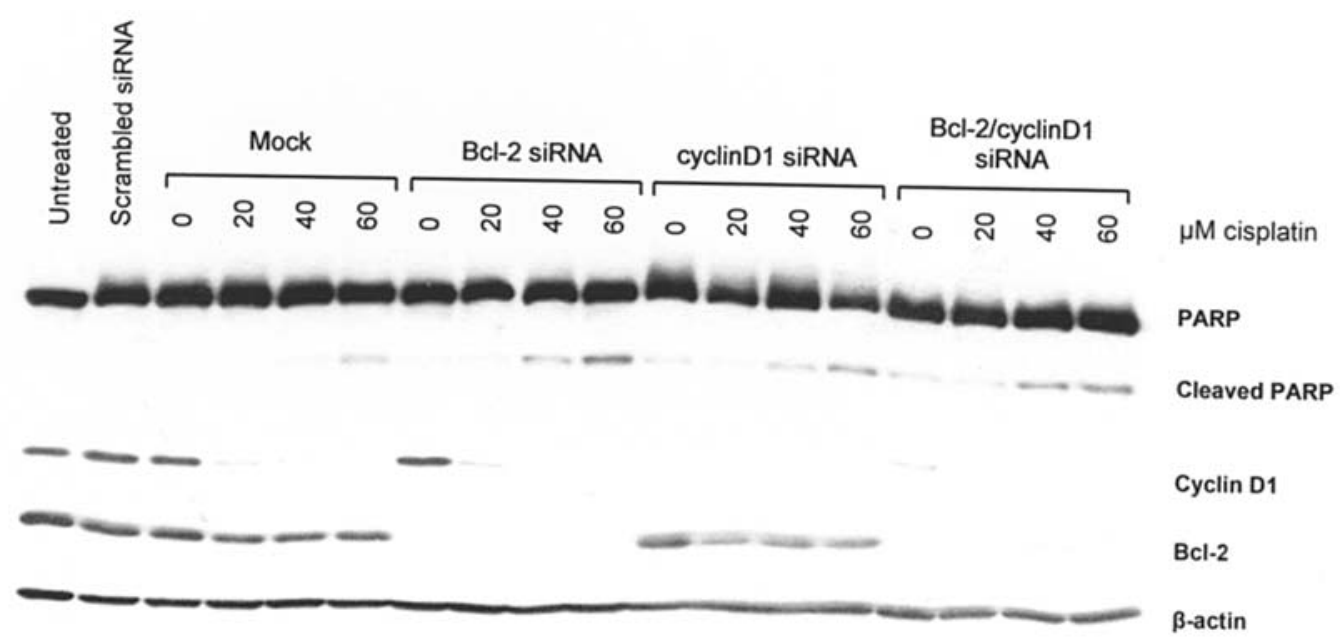

Figure 5. Knockdown of Bcl-2 and cyclin D1 enhances cisplatin-induced PARP cleavage in MCF-7 cells. MCF-7 cells were transfected with siRNA or transfection reagent alone (mock) as described in the legend of Fig. 4 and left for $48 \mathrm{~h}$ before harvesting the cells. The last $24 \mathrm{~h}$ prior to harvesting the cells cisplatin was added to the media of the cells at concentrations indicated. Western blot analysis was performed to measure apoptosis (PARP cleavage) and to detect cyclin D1 and Bcl-2 protein.

Cisplatin treatment is associated with a decrease in cyclin D1 and an increase in cyclin E levels.

Only MCF-7 and CAL-148 cells expressed wild-type p53 which accumulated upon cisplatin treatment, and Ser15 phosphorylation of p53 was seen after cisplatin treatment (Fig. 3). In accordance with the p53 status of the cell lines, up-regulation of the p53-induced protein HDM2 was seen in MCF-7 and in CAL-148 cells (Fig. 3).

A decrease in cyclin D1 levels, accompanied by an increase in cyclin E, was observed in cisplatin-treated cells as compared to untreated cells. Furthermore, it was seen that cisplatin treatment led to increased phosphorylation of ERK1/2, p38 and decreased phosphorylation of PKC. No consistent changes in phosphorylation of GSK3B and Akt were found and no prominent changes could be observed at the protein level of p21, Bcl-2, ERK, p38, Akt, GSK3ß, CK2 or PKC (Fig. 3). It was, however, noted that loss of PTEN in CAL-148 and HCC-1937 cells (Fig. 2) agreed with highly phosphorylated Akt (Fig. 3). In spite of the fact that Akt is known as a pro-survival kinase and has been associated with resistance to drug-induced apoptosis $(27,28)$, we found that a high degree of Akt phosphorylation was not associated with resistance towards cisplatin.

Since cyclin D1 was expressed at barely detectable levels in the most cisplatin sensitive cell line, CAL-148, while expressed at higher levels in the remaining cell lines (Fig. 2) and because a pronounced decrease in cyclin D1 was seen after cisplatin treatment (Fig. 3) cyclin D1 was chosen as the second target for RNA interference studies in MCF-7 cells.

Knockdown of Bcl-2 and cyclin D1 sensitize MCF-7 cells to cisplatin treatment. In order to see if targeting Bcl-2 and/or cyclin D1 would enhance the effect of cisplatin treatment, MCF-7 cells were treated with a pool of Bcl-2 siRNAs, cyclin D1 siRNAs or the two in combination. Evaluating Bcl-2 protein levels 24 and $48 \mathrm{~h}$ after transfection showed that treatment of MCF-7 cells with Bcl-2 siRNA alone resulted in clear reduction of $\mathrm{Bcl}-2$ compared to either of the controls: untreated cells, transfection with a scrambled sequence siRNA (negative control) or with transfection reagent alone (mock) (Fig. 4). The same was true for the level of cyclin D1 in the case of transfection with cyclin D1 siRNA alone (Fig. 4). In the case of siRNA transfection with both Bcl-2 and cyclin D1 siRNA the reduction in Bcl-2 was similar to that observed using Bcl-2 siRNA alone, but the reduction in cyclin D1 was less than with cyclin D1 siRNA alone (Fig. 4) probably caused by the double transfection. It was decided that the reduction of cyclin D1 was sufficient for further studies on the effect on combination of RNA interference with cisplatin treatment.

In mock transfected MCF-7 cells cleavage of PARP was seen after exposure to $60 \mu \mathrm{M}$ of cisplatin while transfection with Bcl-2 siRNA resulted in PARP cleavage using only $40 \mu \mathrm{M}$ of cisplatin (Fig. 5). When cyclin D1 or both Bcl-2 and cyclin D1 were knocked down, PARP cleavage could be observed very slightly even without cisplatin, and when treating these cells with cisplatin much lower doses induced PARP cleavage (20-40 $\mu \mathrm{M})$ as compared to the control (Fig. 5).

We were able to demonstrate an increase in dead/late apoptotic cells using SYTOX green, a nucleic acid staining only permeable for cells that have lost plasma membrane integrity, when MCF-7 cells were transfected with Bcl-2, cyclin D1 or the two in combination compared to mock transfected cells treated with the same amount of cisplatin (Fig. 6A). The fraction of SYTOX positive cells out of total cells was quantified by flow cytometry (Fig. 6B) showing that down-regulation of both Bcl-2 and cyclin D1 enhances the effect of cisplatin and that combining the two targets gives the highest degree of cell death.

Knockdown of cyclin DI results in G1 arrest of MCF-7 cells. Cell cycle analysis was done in order to see if cisplatin or knockdown of Bcl-2 or cyclin D1 could induce a shift in cell cycle distribution. As seen in Fig. 7 no major differences were 

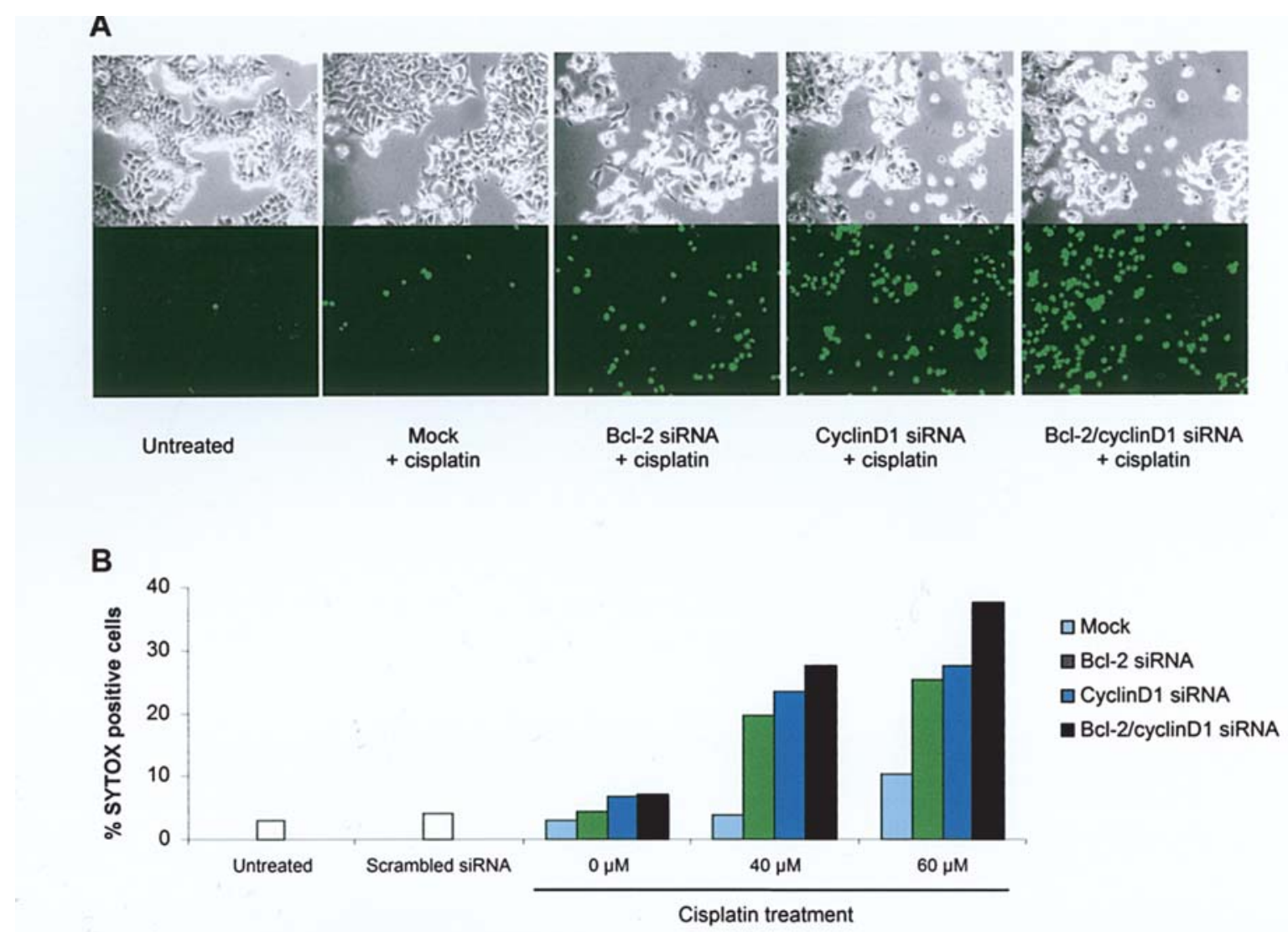

Figure 6. Knockdown of Bcl-2 and cyclin D1 enhances cisplatin-induced cell death in MCF-7 cells. MCF-7 cells were transfected with siRNA and treated with cisplatin as described in the legend of Figs. 4 and 5. Cells were stained with SYTOX green as an indication of cell death. (A) Phase contrast (upper pictures) and fluorescence microscopy (lower pictures) are shown for cells transfected mock-, Bcl-2, cyclin D1 and Bcl-2/cyclin D1 siRNA and treated with $40 \mu \mathrm{M}$ of cisplatin for $24 \mathrm{~h}$. Untreated MCF-7 cell are shown for comparison. Pictures were originally captured at 200 times magnification. (B) SYTOX positive MCF-7 cells were quantified by FACS analysis. Light blue bars represent mock transfected cells, green bars represent Bcl-2 siRNA transfected cells, dark blue bars represent cyclin D1 siRNA transfected cells and black bars represent Bcl-2/cyclin D1 siRNA transfected cells.

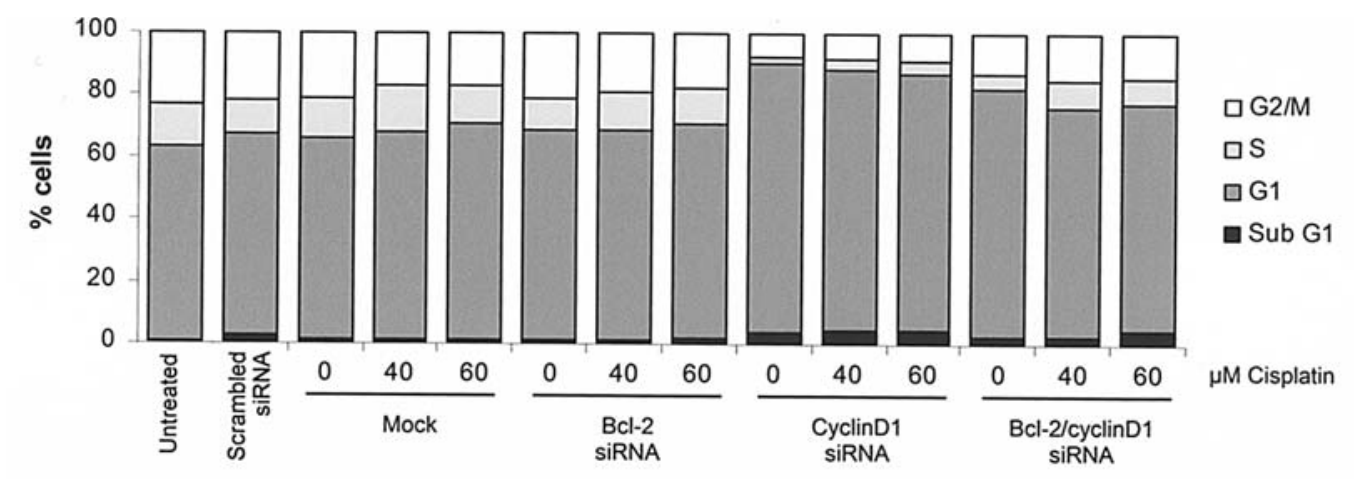

Figure 7. Effect of Bcl-2 and cyclin D1 knockdown and cisplatin treatment on cell cycle distribution of MCF-7 cells. Asynchronously growing MCF-7 cells were transfected with siRNA and treated with cisplatin as described in the legends of Figs. 4 and 5. DNA content was quantified by fixing cells, staining the DNA with propidium iodide and analyzing cells by FACS analysis. Fraction of cells in sub-G1, G1, S and G2/M phases are shown in each bar.

seen in the cell cycle distribution when cell were treated with cisplatin. Similarly, Bcl-2 knockdown did not result in changes in the cell cycle phases. Cyclin D1 knockdown, on the other hand, resulted in a clear increase in the G1phase population and a decrease in both S- and G2/M-phase populations (Fig. 7). Consistent with a less efficient knockdown of cyclin D1 when Bcl-2 and cyclin D1 were knocked down simultaneously (Fig. 4) the increase in the G1-phase population was not as pronounced as in the case of transfection with cyclin D1 alone (Fig. 7). In all cases the fraction of sub-G1phase cells were less than 6\% (Fig. 7), indicating that in our studies cisplatin-induced death of MCF-7 cells was not accompanied by a high degree of DNA fragmentation.

\section{Discussion}

The first part of our study focused on finding two different targets for enhancing the effect of cisplatin on MCF-7 breast 
cancer cells, and in the second part we wanted to show the effect on the cisplatin response by down-regulating these targets, both individually and in combination, using RNA interference.

In our study of five breast cancer cell lines we found that expression of $\mathrm{Bcl}-2$ protein correlated with decreased cisplatin sensitivity. Earlier studies have shown that overexpression of Bcl-2 cells inhibited drug-induced apoptosis in MCF-7 cells $(29,30)$ and that down-regulation of Bcl-2 in MCF-7 cells by siRNA resulted in sensitization of the cells to treatment with etoposide and doxorubicin (31). Therefore, we invetigated whether combining down-regulation of Bcl-2 with targets from different cellular pathways would further sensitize MCF-7 cells to chemotherapy.

When treating different breast cancer cells with cisplatin we found that cisplatin-induced cell death involved a decrease in cyclin D1. Similar changes in cyclin D1 expression were reported by and Li et al (32) after induction of apoptosis in oral cancer cells using 5-fluorouracil (5-FU) and like in our studies on cisplatin the decrease in cyclin D1 was accompanied by an increase in cyclin E. The authors found an accumulation of late G1 and S-phase cells after treatment with 5-FU giving a likely explanation for the observed low levels of cyclin D1 and high levels of cyclin E. Our data, however, could not be explained by a decrease in cyclin D1 expressing early G1-phase cells because cisplatin did not alter the cell cycle distribution in our experiments. Similar results were obtained by Agner et al (33) in human osteocarcinoma cells, where an increase in cyclin $\mathrm{E}$ after treatment with cisplatin was not caused by redistribution of cell cycle phase populations. The authors reported an increased checkpoint kinase 2 (CHK2) activity accompanied by an increase in the level of the E2F-1, a transcription factor stabilized by CHK2 phosphorylation. E2F-1 is known to activate transcription of cyclin $\mathrm{E}$ and therefore this could explain the observed increase in cyclin E. Furthermore, it is known that active GSK3ß phosphorylate cyclin D1, resulting in its degradation (34). Akt phosphorylates GSK3ß at Ser9 and inactivates the kinase. In our analysis, we did not find changes in phosphorylation of Akt (Thr308) or GSK3ß (Ser9) after cisplatin treatment and therefore the decrease in cyclin D1 seems to involve other signals upstream of cyclin D1 rather than the Akt-GSK3ß pathway. The ERK pathway is known to up-regulate cyclin D1 (35), but we showed that cisplatin treatment resulted in activation of ERK. This would be in accordance with an increase in cyclin D1 and not decreased levels as observed. Similarly, Gotoh et al found that neither activity of the Akt-GSK3ß pathway nor the ERK pathway seemed to play a major role in regulating the protein level of cyclin D1 in mouse hepatic tumor cells (36).

We were able to show that cells which already had lowered levels of cyclin D1 due to treatment with cyclinD1 siRNA were more sensitive to cisplatin than normal cells. A crucial question which remains to be answered is how decreased cyclin D1 protein levels result in increased cisplatin-induced apoptosis. We show that lack of cyclin D1 leads to arrest of MCF-7 cells in G1, confirming previous data on knockdown of cyclin D1 (37). This is consistent with the role of cyclin D1 in controlling the G1-S-phase transition, however, it does not explain why MCF-7 cells, where cyclin D1 has been knocked down, are more sensitive to cisplatin than control cells. The answer to this question might be that some of the CDK independent functions of cyclin D1 are in survival pathways. Further studies on these effects will hopefully reveal new exiting aspects of cyclin D1 and perhaps uncover the relationship between cyclin D1 and apoptosis.

In our studies, we could not observe any pronounced cisplatin-induced sub-G1 populations resulting from DNA fragmentation. This is in agreement with other studies and is presumably related to the lack of caspase- 3 in MCF-7 as previously suggested $(38,39)$.

Classically cisplatin is known to cause a transient S-phase arrest followed by a durable G2/M phase arrest (1). Neither S-phase nor G2/M-phase arrest of MCF-7 cells was observed after cisplatin treatment also in agreement with other studies on treatment of MCF-7 cells with cisplatin (39), etoposide and doxorubicin (40). In contrast to drug-induced DNA fragmentation drug-induced G2/M arrest in MCF-7 cells could not be reconstituted by expression of functional caspase-3 (40) indicating that different mechanisms are required for drug-induced DNA fragmentation and G2/M arrest.

In summary, our studies validate the role of Bcl-2 in regulating cisplatin sensitivity in MCF-7 cells and for the first time we show that down-regulation of cyclin D1 sensitize MCF-7 cells to cisplatin. Furthermore, we show novel data indicating that combining two targets, one from the classical apoptotic pathway and the other being a key regulator of cell cycle progression, in this case Bcl-2 and cyclin D1, enhances the effect of cisplatin in $\mathrm{MCF}-7$ breast cancer cells. We have shown that this involves increased cell death, increased cleavage of the caspase target PARP and, in the case of cyclin D1 down-regulation, redistribution of cell cycle populations. It should be noted that even though MCF-7 cells lacks caspase-3, cleavage of PARP still occurs. Caspase-7 has been shown to cleave PARP at the same site as caspase-3 (41) and the observed PARP cleavage in MCF-7 is presumable due to caspase-7 cleavage of PARP.

In prospect of improving future treatment of cancer patients these results look promising in respect to inhibiting multiple cellular survival- and proliferation pathways thereby enhancing the effect of traditional chemotherapy. We have shown that the most efficient killing of MCF-7 cells with cisplatin in our studies was achieved by simultaneous targeting of two different key cell regulators, an antiapoptotic protein and a regulator of cell cycle progression. Based on this knowledge it would be possible to select multiple cellular targets and inactivate them with specific inhibitors in order to improve the chances of efficient tumor treatment. This could pave the way for the design of new sophisticated combination therapies and the chances of optimizing individual patient treatment would surely increase.

\section{Acknowledgements}

This research work was supported by the Danish Cancer Society (grant no. 00252110 9216) and the Natural Science Foundation (grant no. 21-04-0517) to O.G.I. We would like to thank Heidi Wolden-Kirk Graversen for assistance on cyclin D1 knockdown experiments and Barbara Guerra for help with cell cycle analysis. 


\section{References}

1. Siddik ZH: Cisplatin: mode of cytotoxic action and molecular basis of resistance. Oncogene 22: 7265-7279, 2003.

2. Song G, Ouyang $G$ and Bao S: The activation of Akt/PKB signaling pathway and cell survival. J Cell Mol Med 9: 59-71, 2005.

3. Martelli AM, Mazzotti G and Capitani S: Nuclear protein kinase C isoforms and apoptosis. Eur J Histochem 48: 89-94, 2004

4. Litchfield DW: Protein kinase CK2: structure, regulation and role in cellular decisions of life and death. Biochem J 369: 1-15, 2003.

5. Cho HJ, Kim JK, Kim KD, et al: Up-regulation of Bcl-2 is associated with cisplatin-resistance via inhibition of Bax translocation in human bladder cancer cells. Cancer Lett 237: 56-66, 2005.

6. Yang X, Zheng F, Xing H, et al: Resistance to chemotherapyinduced apoptosis via decreased caspase-3 activity and overexpression of antiapoptotic proteins in ovarian cancer. J Cancer Res Clin Oncol 130: 423-428, 2004.

7. Klasa RJ, Gillum AM, Klem RE and Frankel SR: Oblimersen Bcl-2 antisense: facilitating apoptosis in anticancer treatment. Antisense Nucleic Acid Drug Dev 12: 193-213, 2002.

8. Stein CA, Benimetskaya L and Mani S: Antisense strategies for oncogene inactivation. Semin Oncol 32: 563-572, 2005.

9. Raffo A, Lai JC, Stein CA, et al: Antisense RNA down-regulation of bcl-2 expression in DU145 prostate cancer cells does not diminish the cytostatic effects of G3139 (Oblimersen). Clin Cancer Res 10: 3195-3206, 2004.

10. Gillett C, Fantl V, Smith R, et al: Amplification and overexpression of cyclin D1 in breast cancer detected by immunohistochemical staining. Cancer Res 54: 1812-1817, 1994.

11. Massague J: G1 cell-cycle control and cancer. Nature 432: 298-306, 2004.

12. Sherr CJ and Roberts JM: Living with or without cyclins and cyclin-dependent kinases. Genes Dev 18: 2699-2711, 2004.

13. Fantl V, Stamp G, Andrews A, Rosewell I and Dickson C: Mice lacking cyclin D1 are small and show defects in eye and mammary gland development. Genes Dev 9: 2364-2372, 1995.

14. Sicinski P, Donaher JL, Parker SB, et al: Cyclin D1 provides a link between development and oncogenesis in the retina and breast. Cell 82: 621-630, 1995.

15. Yu Q, Geng Y and Sicinski P: Specific protection against breast cancers by cyclin D1 ablation. Nature 411: 1017-1021, 2001.

16. Albanese C, D'Amico M, Reutens AT, et al: Activation of the cyclin D1 gene by the E1A-associated protein p300 through AP-1 inhibits cellular apoptosis. J Biol Chem 274: 34186-34195, 1999.

17. Fu M, Wang C, Li Z, Sakamaki T and Pestell RG: Minireview: cyclin D1: normal and abnormal functions. Endocrinology 145 5439-5447, 2004

18. Warenius HM, Seabra LA and Maw P: Sensitivity to cis-diamminedichloroplatinum in human cancer cells is related to expression of cyclin D1 but not c-raf-1 protein. Int J Cancer 67: 224-231, 1996.

19. Oridate N, Kim HJ, Xu X and Lotan R: Growth inhibition of head and neck squamous carcinoma cells by small interfering RNAs targeting eIF4E or cyclin D1 alone or combined with cisplatin. Cancer Biol Ther 4: 318-323, 2005.

20. Biliran H Jr, Wang Y, Banerjee S, et al: Overexpression of cyclin D1 promotes tumor cell growth and confers resistance to cisplatin-mediated apoptosis in an elastase-myc transgeneexpressing pancreatic tumor cell line. Clin Cancer Res 11: 6075-6086, 2005

21. Maehara Y, Emi Y, Sakaguchi Y, et al: Estrogen-receptornegative breast cancer tissue is chemosensitive in vitro compared with estrogen-receptor-positive tissue. Eur Surg Res 22: 50-55, 1990 .

22. Jänicke RU, Sprengart ML and Wati MR: Caspase-3 is required for DNA fragmentation and morphological changes associated with apoptosis. J Biol Chem 273: 9357-9360, 1998.

23. Saga Y, Mizukami H, Suzuki M, et al: Overexpression of PTEN increases sensitivity to $\mathrm{SN}-38$, an active metabolite of the topoisomerase I inhibitor irinotecan, in ovarian cancer cells. Clin Cancer Res 8: 1248-1252, 2002.
24. Yan X, Fraser M, Qiu Q and Tsang BK: Over-expression of PTEN sensitizes human ovarian cancer cells to cisplatin-induced apoptosis in a p53-dependent manner. Gynecol Oncol 102: 348-355, 2006.

25. Basu A, Weixel K and Saijo N: Characterization of the protein kinase $\mathrm{C}$ signal transduction pathway in cisplatin-sensitive and -resistant human small cell lung carcinoma cells. Cell Growth Differ 7: 1507-1512, 1996.

26. Huang J, Mohanty S and Basu A: Cisplatin resistance is associated with deregulation in protein kinase C-delta. Biochem Biophys Res Commun 316: 1002-1008, 2004.

27. Yang X, Fraser M, Moll UM, Basak A and Tsang BK: Aktmediated cisplatin resistance in ovarian cancer: modulation of p53 action on caspase-dependent mitochondrial death pathway. Cancer Res 66: 3126-3136, 2006.

28. Gagnon V, Mathieu I, Sexton E, Leblanc K and Asselin E: AKT involvement in cisplatin chemoresistance of human uterine cancer cells. Gynecol Oncol 94: 785-795, 2004.

29. Davis JM, Navolanic PM, Weinstein-Oppenheimer CR, et al: Raf-1 and Bcl-2 induce distinct and common pathways that contribute to breast cancer drug resistance. Clin Cancer Res 9: 1161-1170, 2003.

30. Ruiz de Almodovar C, Ruiz-Ruiz C, Munoz-Pinedo C, Robledo G and Lopez-Rivas A: The differential sensitivity of Bc1-2-overexpressing human breast tumor cells to TRAIL or doxorubicininduced apoptosis is dependent on Bc1-2 protein levels. Oncogene 20: 7128-7133, 2001 .

31. Lima RT, Martins LM, Guimaraes JE, Sambade C, Vasconcelos MH: Specific down-regulation of bcl-2 and xIAP by RNAi enhances the effects of chemotherapeutic agents in MCF-7 human breast cancer cells. Cancer Gene Ther 11: 309-316, 2004.

32. Li MH, Ito D, Sanada M, et al: Effect of 5-fluorouracil on $\mathrm{G} 1$ phase cell cycle regulation in oral cancer cell lines. Oral Oncol 40: 63-70, 2004.

33. Agner J, Falck J, Lukas J and Bartek J: Differential impact of diverse anticancer chemotherapeutics on the Cdc25A-degradation checkpoint pathway. Exp Cell Res 302: 162-169, 2005

34. Diehl JA, Cheng M, Roussel MF and Sherr CJ: Glycogen synthase kinase-3beta regulates cyclin D1 proteolysis and subcellular localization. Genes Dev 12: 3499-3511, 1998.

35. Weber JD, Raben DM, Phillips PJ and Baldassare JJ: Sustained activation of extracellular-signal-regulated kinase 1 (ERK1) is required for the continued expression of cyclin D1 in G1 phase. Biochem J 326: 61-68, 1997.

36. Gotoh J, Obata M, Yoshie M, Kasai S and Ogawa K: Cyclin D1 over-expression correlates with beta-catenin activation, but not with H-ras mutations, and phosphorylation of Akt, GSK3 beta and ERK1/2 in mouse hepatic carcinogenesis. Carcinogenesis 24: 435-442, 2003

37. Grillo M, Bott MJ, Khandke N, et al: Validation of cyclin D1/ CDK4 as an anticancer drug target in MCF-7 breast cancer cells: effect of regulated overexpression of cyclin D1 and siRNAmediated inhibition of endogenous cyclin D1 and CDK4 expression. Breast Cancer Res Treat 95: 185-194, 2006.

38. Janicke RU, Sprengart ML, Wati MR and Porter AG: Caspase-3 is required for DNA fragmentation and morphological changes associated with apoptosis. J Biol Chem 273: 9357-9360, 1998.

39. Otto AM, Paddenberg R, Schubert S and Mannherz HG: Cellcycle arrest, micronucleus formation, and cell death in growth inhibition of MCF-7 breast cancer cells by tamoxifen and cisplatin. J Cancer Res Clin Oncol 122: 603-612, 1996.

40. Janicke RU, Engels IH, Dunkern T, Kaina B, Schulze-Osthoff K and Porter AG: Ionizing radiation but not anticancer drugs causes cell cycle arrest and failure to activate the mitochondrial death pathway in MCF-7 breast carcinoma cells. Oncogene 20: 5043-5053, 2001.

41. Germain M, Affar EB, D'Amours D, Dixit VM, Salvesen GS and Poirier GG: Cleavage of automodified Poly (ADP-ribose) polymerase during apoptosis - evidence for involvement of caspase-7. J Biol Chem 274: 28379-28384, 1999. 